\title{
Experimental confirmation of some aspects of the microstructural model of the impedance spectra of porous materials
}

\author{
I. Sánchez ${ }^{1}$, M. Cabeza ${ }^{2}$, M. A. Climent ${ }^{1} \&$ X. R. Nóvoa ${ }^{3}$ \\ ${ }^{I}$ Departament d'Enginyeria de la Construcció, \\ Obres Publiques i Infraestructura Urbana, Universitat d'Alacant, Spain \\ ${ }^{2}$ Dep. Enxeñería dos Materiais, Mecánica Aplicada e Construcción, \\ Universidade de Vigo, Spain \\ ${ }^{3}$ Dep. Enxeñería Química, Universidade de Vigo, Spain
}

\begin{abstract}
The use of impedance spectroscopy, IS, has become a useful technique for the microstructural characterisation of cement pastes and mortars. The main advantage is that it is non destructive, but in the other hand the interpretation of the results obtained is not straightforward. A model has been proposed that gives microstructural interpretation of the electrical parameters obtainable from the impedance data. Here is presented a validation of that model, using a model material, which allows controlled changes in the electrolyte conductivity to be monitored by IS. The conductivity of pore solution cannot be controlled in cementitious materials, which makes very difficult to differentiate between changes of pore structure and changes in conductivity. The results presented here show good agreement between predictions of the model, and experimental results.
\end{abstract}

Keywords: microstructure, impedance spectroscopy, conductivity, modelling.

\section{Introduction}

The study of the microstructure of concrete and mortar is of great interest for civil engineers because the pore network is directly related to the mechanical properties of this material, and also to the durability.

There are several techniques for determining the pore structure of a material [1], but most of them are destructive and cannot be used in situ. In the 90s 
impedance spectroscopy was used to study in a non destructive way the microstructure of cementitious materials [2,3]. This technique can also be used in situ in real structures, and the measurements are fast and easy to perform. The main drawback is the accurate interpretation of the measurements obtained. Nowadays it is well established that in each impedance spectra of cement pastes, mortars and concrete two time constants are present [4]. Proper equivalent electrical circuits involving two time constants have been proposed for the interpretation of the impedance spectra. Those models have been tested in different experimental conditions, with good agreement with the hypothesis established [4]. The relationship between the electrical parameters (resistances and capacitances) and the microstructure of the system (solid paste and aggregates, electrolyte, conductivity, pore dimensions and pore surface) was established in a recent model [5]. That work states that the unreacted solid phase is the responsible of the high frequency capacitance in the impedance spectra, while the low frequency capacitance accounts for the solid-electrolyte interface in the filled pores. The equivalent resistance is dependent on the geometry of pores and also on the conductivity of the inner electrolyte. The model was checked, with good results, with the impedance spectra of different ordinary Portland cement pastes and mortars.

For those materials it is not possible to split the effects of conductivity and pore dimensions. In ordinary Portland cement paste the value of electrolyte conductivity can be assumed to be constant, but in the case of cements with pozzolanic activity the value of the conductivity may vary because of the pozzolanic reactions that consume portlandite to form new C-S-H phases, with the consequent changes in the composition of the electrolyte. Obviously, changes in the pore dimensions also occur because of those new solids formed at pore walls.

The model has already been used to interpret microstructural modifications in pozzolanic cement mortars [5], but the conductivity was supposed to remain constant in the interpretation of those results. So, it seems to be very important to be able to study the influence of the conductivity by itself on the dielectric response with no variation of the pore structure, as a first step.

One of the objectives of this work is to set an experimental procedure involving samples of controlled electrolyte conductivity inside the pores. The second step is to study the influence of just electrolyte conductivity on the impedance spectra of a porous material.

The experimental section is devoted to describe the procedure followed to have a sample with known and controlled conductivity in the pores, and in the results section the impedance data and the simulations are compared.

\section{Experimental setup}

\subsection{Sample preparation}

In order to validate the electrical equivalent model taking into account the contribution of the conductivity of pore solution, a commercial ceramic material was chosen. The mineralogical composition of the material was determined to be 
cordierite, and the pore structure was also very simple, as will be shown later. The thickness of the sample was of $0.5 \mathrm{~cm}$.

This material mainly was chosen because no leaching of ions from the matrix is expected. All the pores of the material were first saturated with distilled water, using the saturation procedure described in the ASTM standard 1202-97 [7]. After saturation the sample was boiled for 4 hours in distilled water and the conductivity was measured before and at the end of the experiment, with no significant changes in the conductivity, meaning no leaching from the ceramic.

This result meant that the conductivity can be controlled by preparing solutions of different salt concentration, and saturating the empty pores with the chosen solution. The solutions were prepared using $\mathrm{KCl}$ (because of the equal mobility of both ions) to get $0.01,0.05,0.1$ and $0.5 \mathrm{M} \mathrm{KCl}$ solutions. The conductivity of each solution was measured, and the results are shown in Figure 1.

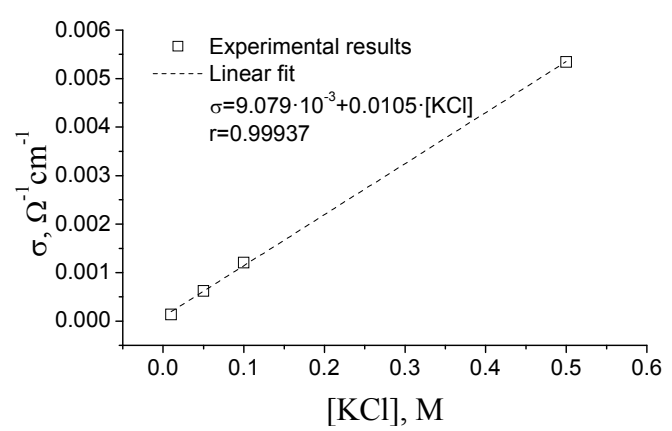

Figure 1: Values of conductivity for the different solutions of $\mathrm{KCl}$ used to fill the pores of the ceramic material. Conductivity values were taken at a temperature of $25^{\circ} \mathrm{C}$.

The procedure for having the sample with all the pores saturated with a solution of known conductivity is the same procedure as that for saturating the pores with distilled water. The sample used was initially vacuum-dried, to ensure that all the pores are empty. After the time necessary for drying the sample, it was saturated by immersion in the corresponding solution and keeping it in vacuum (about $1 \mathrm{mmHg}$ ) for 24 hours, so that the solution will fill the pores and remaining air will be released. Impedance spectra were recorded on saturated samples.

After the impedance spectroscopy measurement, and in order to remove the filling solution, the sample was boiled again in distilled water for three hours. The saturation procedure was then restarted using fresh solution. The saturation was made with the solutions in order of increasing concentration, or increasing conductivity. This fact is important because, for a given experiment, even though not all the ions of the preceding experiment were removed, their influence in the current conductivity measurement is negligible. 


\subsection{Mercury intrusion porosimetry measurement}

The pore structure of the material is also a key parameter into the model because the total porosity and the pore size distribution are employed in simulations.

Mercury intrusion porosimetry was used to characterise the pore structure of the material. The equipment employed was an AUTOPORE IV 9500 from Micromeritics. This porosimeter allows pore diameter determination in the range from $5 \mathrm{~nm}$ to $0.9 \mathrm{~mm}$. The total porosity measured was $22 \%$ in volume, and the pore size distribution is shown in Figure 2. Only one pore family, with centre at about $3900 \mathrm{~nm}$, was found.

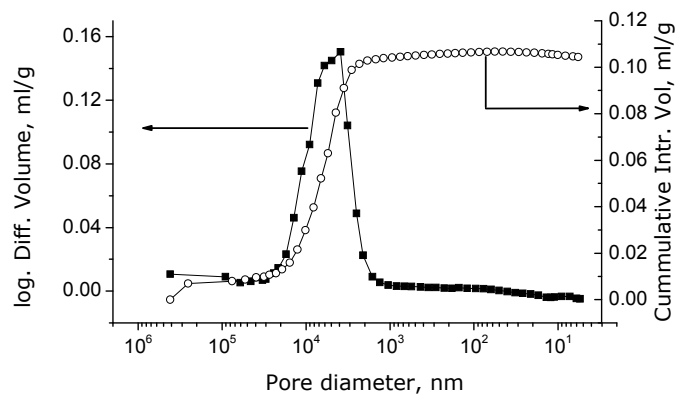

Figure 2: $\quad$ Pore size distribution of the ceramic material used.
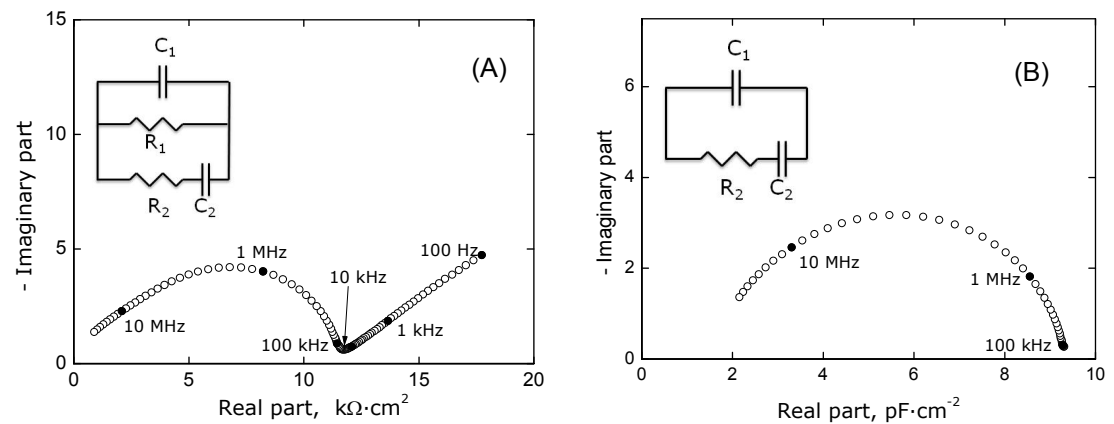

Figure 3: Impedance spectra of the ceramic material used with a solution $0.01 \mathrm{M}$ of $\mathrm{KCl}$ filling the pores. (A) corresponds to the contacting method, and (B) corresponds to the Cole-Cole representation, using the non contacting method. The equivalent circuits used for the fitting of the impedance spectra are also shown on each figure.

It is well known that only the pore diameter at the open surface of the sample is measured using this technique, as described in the literature [8]. This fact is not important at all. The model includes a major simplification of the pore structure, but it represents in an appropriate way the reality of complex materials [5]. 


\subsection{Impedance spectroscopy measurements}

The impedance spectra were obtained in a frequency range from $40 \mathrm{MHz}$ down to $100 \mathrm{~Hz}$. The impedance analyzer used was a HP4194A, which allows measurements in capacitance range from $10^{-14} \mathrm{~F}$ to $0.1 \mathrm{~F}$, with a maximum resolution of $10^{-15} \mathrm{~F}$. The electrodes were of flexible graphite, attached to copper plates of $4 \mathrm{~cm}$ diameter. Measurements were made both with contact between electrode and sample, and with a foil of polymer interposed between the graphite sheet and the sample, to minimise double layer effects, as explained in previous works $[3,4]$.

The recorded impedance spectra are very similar to those obtained for cementitious materials, and hence the equivalent circuits used for the interpretation are the same. Figure 3 depicts an example of the impedance spectra measured, together with the corresponding equivalent circuit.

The equation corresponding to that circuit is the Havriliak-Negami equation and includes all the electric elements present in the circuit, and the symmetric and asymmetric dispersion factors, $\alpha$ and $\beta$.

$$
C(f)=C_{1}+\frac{C_{1}}{\left(1+\left(2 \pi f \cdot R_{2} \cdot C_{2}\right)^{\alpha}\right)^{\beta}}
$$

\subsection{Simulations}

The simulations were performed using MATLAB ${ }^{\odot}$. The model proposed and the calculations have been reported elsewhere [5]. The model is based in the transmission line model proposed by Park and McDonnald [9]. The simulations were performed considering only one pore family, with dispersion around a central value of $4 \mu \mathrm{m}$, approximately the central pore value obtained from MIP for the material employed. The values for the conductivity of the electrolyte were different but within the same range of variation. The total porosity used in the simulations was of $22 \%$, in coincidence with the total porosity of the material. The spectra were obtained under the hypothesis of no contact among electrode and sample. The spectra obtained of the simulations were fitted using the same equivalent circuit. The results of the fitting of the simulations will be compared with the experimental results as a function of the electrolyte conductivity. The scheme of the model proposed for the calculations is shown in [5].

\section{Results and discussion}

The impedance spectroscopy data were fitted using the equivalent circuits above presented. The results of the fitting as a function of the conductivity are presented here. Initially the results presented correspond to the non contacting method, with the plastic foil between electrode and sample.

The value of the capacitance $C_{1}$ remains essentially constant for the experimental results, as expected, and is defined as constant for the simulations. The results for the capacitance associated to the solid phase are shown in Figure 4. 
Figure 5 depicts the best fitting values obtained for the low frequency parameters, $R_{2}$ and $C_{2}$. In that figure the results predicted by the model are also presented. The measurements were made with four different values of conductivity in the pore network of the material, and the simulations were made using 5 different points in the same range of variation of conductivity. As it can be seen in these figures, for both real measurements and simulations the behaviour of the parameters is the same. The capacitance $\mathrm{C}_{2}$ has no clear tendency and remains almost constant while the resistance decreases in a double logarithmic way as the conductivity of the solution inside the pores increases.

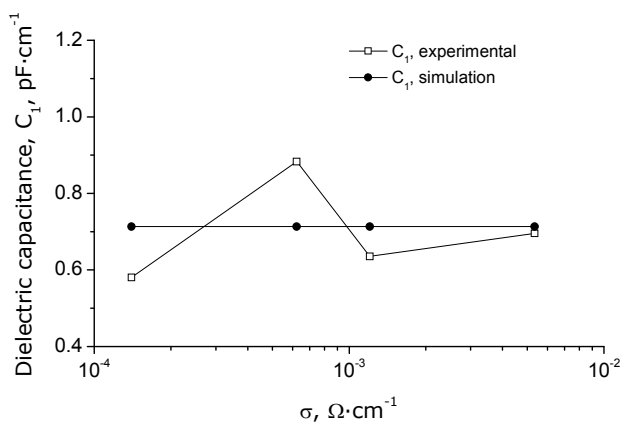

Figure 4: Dielectric capacitance, calculated using the model (solid circles) and obtained from the impedance measurements in the ceramic material, for different values of electrolyte conductivity.

As it can be seen in Figure 6, at low conductivity of the electrolyte the symmetric dispersion factor $\alpha$ is close to 1 , while the dominant is the asymmetric dispersion factor $\beta$, whose value is about 0.7 , meaning asymmetry in the impedance spectra. When the conductivity is increased, the value of the asymmetric dispersion factor tends to 1 (meaning no asymmetry in the impedance spectra) and the value of the symmetric dispersion factor decreases, meaning a more significant symmetrical dispersion in the impedance spectra.

The values of the capacitances $\mathrm{C}_{1}$ and $\mathrm{C}_{2}$ remain almost constant with conductivity changes. This result is in agreement with the model predictions. The capacitance $\mathrm{C}_{1}$ is associated to the solid phase fraction in the material. Changes in the conductivity of the electrolyte do not involve any change in the solid phase. The experimental results as shown in Figure 4 do not show a clear tendency and only show not important changes around a central value. This central value, as expected is the prediction of the model for the capacitance $\mathrm{C}_{1}$.

The value of the low frequency capacitance $C_{2}$ is associated with the solidelectrolyte interface inside pores. Changes in the surface (due, i.e. to new solids formed at the pore walls) will be clearly reflected by this capacitance [6]. As stated before, no solids where formed in our system, and no part of the ceramic matrix was destroyed, hence no changes are expected in the surface of pore walls and thus $\mathrm{C}_{2}$ shall remain constant. 
One reason that could imply a change in the solid-electrolyte contact surface is salt precipitation at the pore walls during the drying period. The experimental procedure, involving boiling in distilled water for about 3 hours, seems to be good enough to remove every rest of possible salt precipitate inside the pores. Both experimental and simulation data show negligible fluctuations on $\mathrm{C}_{2}$ capacitance values. These results agree with the physical meaning of this parameter previously stated.
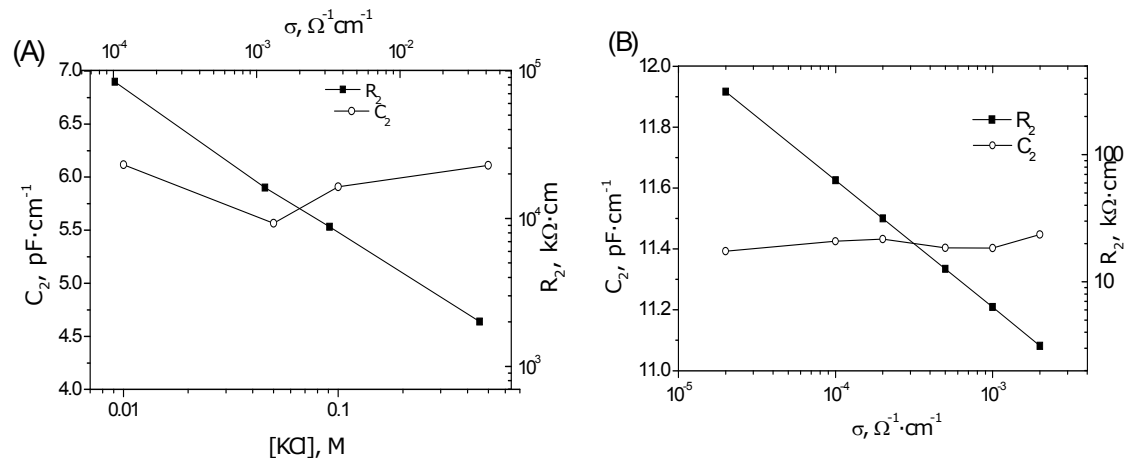

Figure 5: Dependence of the parameters $R_{2}$ and $C_{2}$ with the conductivity of the electrolyte in the experimental measurements $(\mathrm{A})$ and in the impedance spectra obtained using the theoretical model (B).

The model also could be used to explain the dispersion factors in the dielectric response of the materials. The evolution of these factors with the conductivity value is shown in Figure 6.

The value of the pore resistance changes with the electrolyte conductivity, as expected. This result is obvious because of the inverse relationship between conductivity and resistivity, but it also confirms that the association of this resistance with the electrolyte in the pores is right. The value of the resistance decreases as the value of the conductivity of the electrolyte (or ionic concentration) increases, as depicted in Figure 7. This factor has to be taken into account when dealing with cements with pozzolanic activity, especially when a high portion of clinker is substituted by a material with pozzolanic activity. The pozzolanic reactions may change the conductivity of the electrolyte, and these possible variations should be taken into account in the interpretation of the impedance spectra. The changes in the resistance maybe only attributed to the changes in pore size and this interpretation can be erroneous.

According to the model, $\mathrm{R}_{1}$ accounts for the connectivity of percolating pores while $\mathrm{R}_{2}$ non-percolating pores. The interesting point here is the dissimilar dependence with electrolyte concentration between $R_{1}$ and $R_{2}$ depicted in Figure 7. While $\mathrm{R}_{2}$ varies in agreement with the conductivity data reported in Figure 5 the slope for $\mathrm{R}_{1}$ dependence is higher, which suggest the formation of new percolating paths as the electrolyte concentration increases. The associated mechanism can be additional ionic flux due to osmotic pressure. 


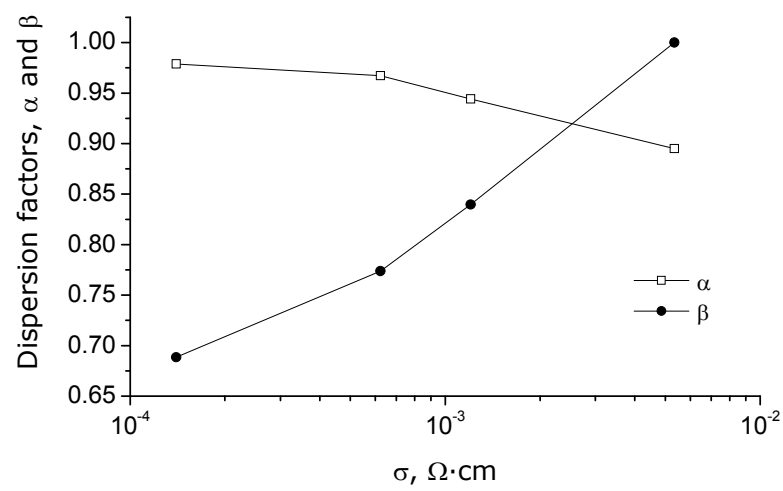

Figure 6: Evolution with conductivity of the symmetric and asymmetric dispersion factors in the impedance spectra of the ceramic material (Eq. 1).

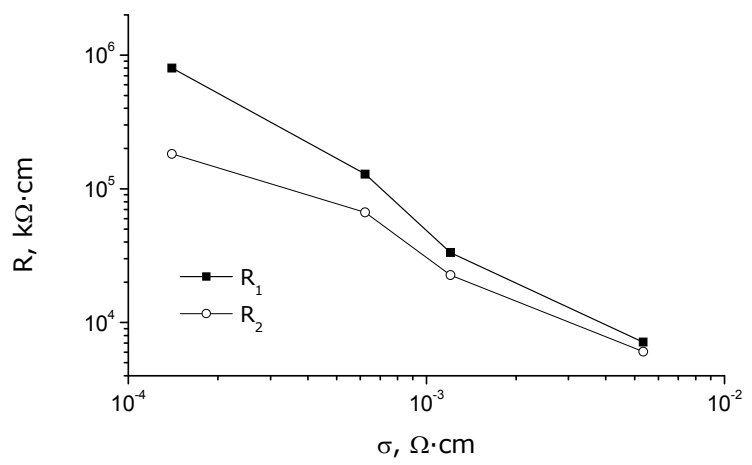

Figure 7: $\quad$ Evolution for different values of conductivity of the resistances $\mathrm{R}_{1}$ and $R_{2}$ in the measurements made with contact between the electrodes and the sample.

Finally the values of the dispersion factors need some discussion. Under the experimental procedure followed, with the pores of the material saturated with the electrolyte, all the pores contribute to the measurement, and there is only one pore family present. Under those conditions, as it has been demonstrated in [5] only the symmetric dispersion factor will have a value lower than 1 , and the asymmetric dispersion factor should be constant and equal to 1. Figure 6 shows a behaviour different than predicted taking into account the pore dimensions and structure. To understand the behaviour of these parameters, the penetration depth of an AC signal into a pore has to be taken into account. The penetration depth can be written as

$$
\delta=\left(\frac{Z_{1}+Z_{2}}{Z_{3}}\right)^{1 / 2}
$$


for a transmission line as shown in Figure 8:, used for the modelling of the dielectric response of porous materials [5].

The value of the penetration depth depends, among other factors, on the conductivity of the electrolyte included in $Z_{2}$ in the model. The influence of the conductivity is shown in Figure 9. It can be seen that, at a given frequency, the value of the penetration depth increases significantly as conductivity does. This result is predicted by the model. Thus, the frequency range at which the AC signal penetrates the sample is increased when the conductivity of the electrolyte increases. According to Figure 9 , at $10^{4} \mathrm{~Hz}$ the electric signal is not able to penetrate the sample for $\sigma_{0}=1 \cdot 10^{-4} \Omega^{-1} \mathrm{~cm}^{-1}$, but it penetrates up to $0.9 \mathrm{~cm}$ when the conductivity is increased by 100 times.

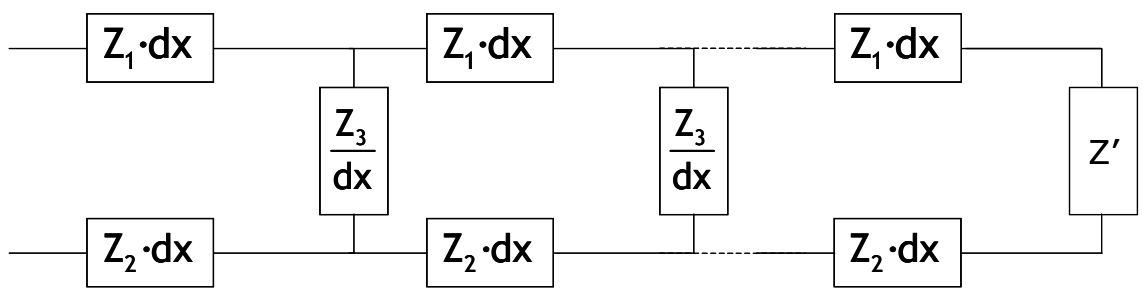

Figure 8: Transmission line model used as a base of the model, and also used to compute the penetration depth.

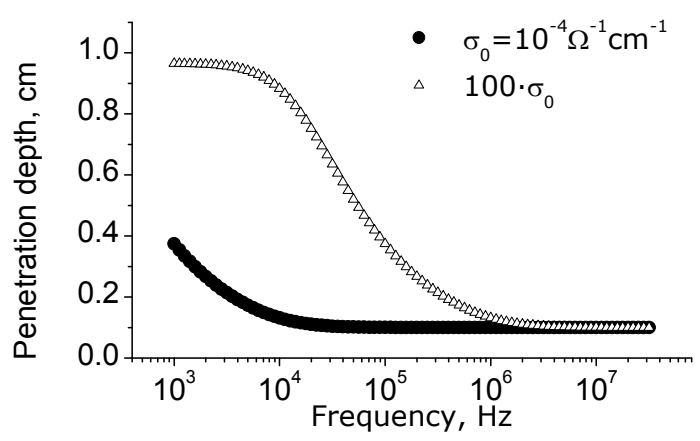

Figure 9: Changes in penetration depth (numerical calculations) for different values of electrolyte conductivity. Sample thickness was of $0.5 \mathrm{~cm}$.

The low penetration of the signal due to low conductivity of the electrolyte causes asymmetric dispersion in the time constant [5], and justifies that with the solution of lower content in $\mathrm{KCl}$ the dielectric response is asymmetrically shaped, with a $\beta$ value about 0.7 . When de conductivity increases the effect of the penetration depth disappears, and symmetrical distribution of pore sizes is reflected by the impedance spectra with a factor $\alpha$ close to 0.85 , as it could be expected for a pore size distribution such as that present in this material. 
This fact of the asymmetrical dispersion due to the conductivity is not so clear in materials with a more complex pore size distribution such as cement pastes or mortars. In those materials the differences in pore size can also be responsible of the asymmetry of the time constant, and will not clearly reflect a lower value of conductivity due to portlandite consumption in the possible pozzolanic reactions.

\section{Conclusions}

From the study presented we can conclude that an experimental procedure has been established for the saturation of the pores of a material with different electrolytes, in order to control the conductivity. The model previously proposed is accurate for the study of the dielectric response of porous materials. Results are in good agreement for materials with a complex pore network and also for much simpler materials.

The effect of the electrolyte conductivity has to be taken into account because it has a huge influence on the resistance associated to the pores. This factor also affects the symmetry of the dispersion in time constants present, but this effect will not be so clear in materials with a great range of pore diameters.

\section{Acknowledgements}

Authors would like to thank by the financial supported the Ministerio de Educación y Ciencia of Spain and Fondo Europeo de Desarrollo Regional (FEDER) through project BIA2006-05961.

\section{References}

[1] E. Robens B. Benzler, G. Büchel, H. Reichert, K. Schumacher; Investigation of characterizing methods for the microstructure of cement. Cement and Concrete Research, (32) (2002) 87-90

[2] W.J. McCarter, R. Brousseau; The a.c. response of hardened cement paste, Cem. Concr. Res. 20 (1990) 891-900

[3] M. Keddam, H. Takenouti, X.R. Nóvoa, C. Andrade, C. Alonso; Impedance measurements on cement paste, Cem. Concr. Res. 27 (1997) 1191- 1201.

[4] M. Cabeza, P. Merino, A. Miranda, X.R. Novoa, I. Sanchez; Impedance spectroscopy study of hardened Portland cement paste. Cem. Concr. Res. 32 (2002) 881-891

[5] M. Cabeza, M. Keddam, X.R. Nóvoa, I. Sánchez, H, Takenouti; Impedance spectroscopy to characterize the pore structure during the hardening process of Portland cement paste. Electrochim. Acta, 51(2006) 1831-1841

[6] I. Sánchez, M.P. López, M.A. Climent; Effect of Fly Ash on Chloride Transport through Concrete: Study by Impedance Spectroscopy; Proceedings of the 12th International Congress on the Chemistry of Cement; edited by J.J. Beaudoin, J.M. Makar and L. Raki; Durability and Degradation of Cement Systems: Corrosion and Chloride Transport; T4.044; published by the National Research Council of Canada; Montreal Canada; (2007). ISBN: 978-0-660-19695-4 
[7] ASTM Standard C 1202-97: standard test method for electrical indication of concrete's ability to resist chloride ion penetration. Annual book of ASTM Standard Section 4 Vol 04.02 (2000)

[8] S. Diamond; Mercury porosimetry. An inappropriate method for the measurement of pore size distributions in cement-based materials. Cement and Concrete Research. 30 (2000) 1517-1525

[9] J.R. Park, D.D. McDonnald; Impedance Studies of the growth of porous Magnetite films on carbon steel in high temperature aqueous systems. Corrosion Science 23 (1983) 295-315 